\title{
Lymph node ratio is an important and independent prognostic factor for patients with stage III melanoma
}

\author{
Adam C. Berger \\ Kimmel Cancer Center, Thomas Jefferson University, Philadelphia, PA, adam.berger@jefferson.edu \\ Michael Fierro \\ Kimmel Cancer Center, Thomas Jefferson University, Philadelphia, PA \\ John C. Kairys \\ Kimmel Cancer Center, Thomas Jefferson University, Philadelphia, PA, john.kairys@jefferson.edu \\ David Berd \\ Kimmel Cancer Center, Thomas Jefferson University, Philadelphia, PA, David.Berd@jefferson.edu \\ Fakraminfistạd additional works at: https://jdc.jefferson.edu/kimmelccfp \\ Kimmel Cancer Center, Thomas Jefferson University, Philadelphia, PA, Takami.Sato@jefferson.edu \\ Part of the Dermatology Commons, Oncology Commons, and the Surgery Commons

\section{Let us know how access to this document benefits you} \\ See next page for additional authors
}

\section{Recommended Citation Papers. Paper 8. \\ https://jdc.jefferson.edu/kimmelccfp/8}

Berger, Adam C.; Fierro, Michael; Kairys, John C.; Berd, David; Sato, Takami; Andrel, Jocelyn; Hyslop, Terry; and Mastrangelo, Michael J., "Lymph node ratio is an important and independent prognostic factor for patients with stage III melanoma" (2012). Kimmel Cancer Center Faculty

This Article is brought to you for free and open access by the Jefferson Digital Commons. The Jefferson Digital Commons is a service of Thomas Jefferson University's Center for Teaching and Learning (CTL). The Commons is a showcase for Jefferson books and journals, peer-reviewed scholarly publications, unique historical collections from the University archives, and teaching tools. The Jefferson Digital Commons allows researchers and interested readers anywhere in the world to learn about and keep up to date with Jefferson scholarship. This article has been accepted for inclusion in Kimmel Cancer Center Faculty Papers by an authorized administrator of the Jefferson Digital Commons. For more information, please contact: JeffersonDigitalCommons@jefferson.edu. 


\section{Authors}

Adam C. Berger, Michael Fierro, John C. Kairys, David Berd, Takami Sato, Jocelyn Andrel, Terry Hyslop, and Michael J. Mastrangelo 


\section{As submitted to: \\ Journal of Surgical Oncology}

And published as:

Lymph node ratio is an important and independent prognostic factor for patients with stage III melanoma

\section{Volume 105, Issue 1, January 2012, Pages 15-20 \\ DOI: $10.1002 /$ jso.22051}

Adam C. Berger, MD

Michael Fierro, MD

John C. Kairys, MD

David Berd, MD

Takami Sato, MD, PhD

Jocelyn Andrel, MSPH, MS

Terry Hyslop, PhD

Michael J. Mastrangelo, MD

Berger, A. C., Fierro, M., Kairys, J. C., Berd, D., Sato, T., Andrel, J., et al. (2012).

Lymph node ratio is an important and independent prognostic factor for patients with stage III melanoma. Journal of Surgical Oncology, 105(1), 15-20. 


\section{LYMPH NODE RATIO IS AN IMPORTANT AND INDEPENDENT}

\section{PROGNOSTIC FACTOR FOR PATIENTS WITH STAGE III MELANOMA.}

\author{
Adam C. Berger ${ }^{1}$, MD \\ Michael Fierro ${ }^{1}$, MD \\ John C. Kairys ${ }^{1}$, MD \\ David Berd ${ }^{2}$, MD \\ Takami $\mathrm{Sato}^{2}, \mathrm{MD}, \mathrm{PhD}$ \\ Jocelyn Andrel, MSPH ${ }^{3}$, MS \\ Terry Hyslop ${ }^{3}, \mathrm{PhD}$ \\ Michael J. Mastrangelo ${ }^{2}$, MD
}

From the Departments of Surgery ${ }^{1}$ and Medical Oncology ${ }^{2}$ and the Division of Biostatistics ${ }^{3}$, Kimmel Cancer Center, Thomas Jefferson University, Philadelphia, PA 19107

Correspondence: Adam C. Berger, MD; 1100 Walnut Street, MOB, Suite 500;

Philadelphia, PA 19107; phone-(215) 955-1622; fax-(215) 923-8222; email: adam.berger@jefferson.edu

Poster Presentation at the Society of Surgical Oncology Cancer Symposium; Phoenix, AZ; March 2009.

Running Title: Lymph node ratio in stage III melanoma

| Key Words: melanoma, lymph node dissection, lymph node ratio

Deleted: MALIGNANT

MELANOMA

\section{ABSTRACT}


Introduction: The incidence of melanoma is dramatically increasing worldwide. We hypothesized that the ratio of metastatic to examined lymph nodes (LNR) would be the most important prognostic factor for stage III patients.

Methods: We retrospectively reviewed our institutional database of melanoma patients and identified 168 patients who underwent lymph node dissection (LND) for stage III disease between 1993 and 2007. Patients were divided into 3 groups based on LNR $(\leq 10 \%, \mathrm{n}=93 ; 10-\leq 25 \%, \mathrm{n}=45 ;$ and $>25 \%, \mathrm{n}=30)$. Univariate and multivariate analysis was performed using Cox proportional hazards model.

Results: The median survival time of the entire group of patients was 34 months. The median number of positive nodes was 2 (range $=1,55)$, and the median number of examined nodes was 22 (range $=5-123$ ). Tumor characteristics of the primary melanoma (such as thickness, ulceration, and primary site) were not significant predictors of survival in this analysis. By univariate analysis, LNR was an important prognostic factor. Patients with LNR 10-25\% and $>25 \%$ had decreased survival compared to those patients with $\mathrm{LNR} \leq 10 \%(\mathrm{HR}=$ hazard ratio $=2.0$ and 3.1, respectively; $\mathrm{p} \leq 0.005)$. The number of positive lymph nodes also impacted on survival ( $\mathrm{p}=0.001)$. In multivariate analysis, LNR of $10-25 \%$ and $>25 \%$ predicted survival ( $\mathrm{HR}=2.5$ and 4.0 , respectively).

Conclusion: LNR is an important prognostic factor in patients undergoing LND for stage III melanoma. It can be used to stratify patients being considered for adjuvant therapy trials and should be evaluated using a larger prospective database. 
Berger-3 


\section{INTRODUCTION}

Between the years of 1975 and 2005 the annual incidence in melanoma in the United States increased from 7.9 per 100,000 to 21.5 per 100,000, while the death rate has increased from 2.1 to 2.7 per $100,000^{1}$. It has been estimated that in fair-skinned populations the incidence is currently increasing by $3-7 \%$ per year worldwide.

Stage III melanoma is defined by the metastatic infiltration of 1 or more lymph nodes or by intra-lymphatic metastasis in the form of in-transit or satellite lesions. These criteria represent a heterogenous group of individuals with 5-year survival ranging from $69 \%$ to as low as $13 \% .^{2}$ Prognostic indicators which are used for TNM staging of stage III patients include the number of metastatic lymph nodes, the tumor burden within positive nodes (microscopic or macroscopic), the presence or absence of ulceration in the primary lesion, and the presence of in-transit or satellite metastasis.

Due to the high toxicity and variable response to adjuvant treatments for melanoma, it is essential to establish new prognostic indicators which can be used to direct the management of Stage III melanoma patients. While biologic and genetic markers have been identified, they are not yet available for widespread use. Thus there is a call for new, readily available clinical prognostic indicators. One factor which is consistently shown to be an important determinant of survival in stage III patients is increasing number of positive lymph nodes. ${ }^{3,4}$ Another prognostic factor which has become more prominent in many malignancies is the ratio of positive to total examined lymph nodes or lymph node ratio (LNR). ${ }^{5,6}$ 
There is recent evidence that LNR is an important prognostic factor in malignancies such as gastric cancer, esophageal cancer, and breast cancer. Two recent reports from Japan and Spain have demonstrated that increasing LNR is a significant prognostic indicator for worse survival in gastric cancer ${ }^{7,8}$. A recent publication also demonstrated the importance of LNR in pancreatic cancer ${ }^{9}$. Finally, two recent studies Deleted: a Deleted: y Deleted: malignant melanoma demonstrated the importance of LNR in patients with melanoma undergoing radical Formatted: Superscript lymph node dissection 5,10 . In this study we hypothesize that the ratio of metastatic lymph nodes to total nodes excised is an independent prognostic indicator of survival in Stage III melanoma patients. 


\section{METHODS}

After obtaining IRB approval, the Tumor Registry at Thomas Jefferson Hospital was queried to identify all patients who underwent lymph node dissection (LND) for stage III melanoma. Two-hundred sixteen patients were identified who met these criteria

Deleted: malignant melanoma for the period of August 1993 to May 2007, and 168 patients had complete data (for most variables), and comprise our study cohort In this study, 93 patients (55\%) underwent therapeutic LND based on clinical findings, 14 (8\%) received prophylactic dissection, and $61(36 \%)$ had a positive sentinel lymph node biopsy (SNB).

A three-level axillary lymph node dissection was most commonly employed $(\mathrm{n}=112,52 \%)$. A total of 68 patients $(40 \%)$ underwent some combination of inguinal and/or iliac dissection. Eleven patients underwent cervical lymph node dissection alone (7\%) and 5 patients had a combination of cervical and axillary dissection. After dissection, all patients were followed with physical examination, history, blood work, and imaging which included alternating chest x-rays and cross-sectional imaging. Follow-up was generally every 3 months for the first two years and then every 6 months thereafter. The median follow-up for the entire group was 26 months (range 0.5-152 months).

The following clinical and pathologic variables were collected for univariate and multivariate analysis: age $(<50, \geq 50)$, sex, year of surgery, site of primary tumor (head/neck, trunk, extremity), tumor thickness $(\leq 1,1-\leq 2,2-<4, \geq 4)$, ulceration (yes, no, or unknown), type of adjuvant treatment (vaccine, chemotherapy meds, IFM, combination, none/unknown), lymph node basin dissected (axillary, inguinal, iliac, 
cervical, or combination), number of positive nodes (1, 2-3, $\geq 4$ - AJCC stages N1, N2, and $\mathrm{N} 3$ respectively) and the ratio of histologically positive lymph nodes to total number of nodes examined - the Lymph Node Ratio (LNR) (The total number of excised nodes (continuous; and also categorical: $<20,21-40,>40$ ) are also presented in univariate analysis). The lymph node ratio was categorized into 3 different groups based on the paper by Rossi et al $^{5}--\leq 10 \%$ (group 1), $10-<25 \%$ (group 2), and >25\% (group 3). Survival was calculated from the date of lymph node dissection to date of last follow-up or death. Patients were staged according to the AJCC $6^{\text {th }}$ edition, although the majority of patients did undergo dissection prior to $2002(\mathrm{n}=122,73 \%)$ when those guidelines were published.

Survival curves were generated using the Kaplan-Meier method. Univariate and multivariate analysis were preformed with the Cox proportional hazards model. To determine the best variable to assess lymph node involvement, 3 separate multivariate models were fit, one with number of positive lymph nodes, one with lymph node ratio alone, and one model with both of these variables. The Akaike Information Criterion, which is a measure of the goodness of fit of an estimated statistical model, was used to select the preferred model 11 . Results with $P$ values of less than 0.05 were considered 


\section{RESULTS}

The average age of patients at time of LND was 57.9 years old (range $=18-86$ ); there were 103 males and 65 females (ratio $=1.6: 1)$. Primary tumors were most commonly located on the trunk $(\mathrm{n}=86,51 \%)$, followed by upper or lower extremity $(\mathrm{n}=$ $74,44 \%)$, and head and neck $(n=8,5 \%)$, No patients had an unknown primary site. The clinicopathologic characteristics from this series are presented in Table 1. The majority of patients were 50 years or older at the time of dissection $(n=116,69 \%)$. Most patients had original primary tumors which were of intermediate thickness-between 1 and $4 \mathrm{~mm}$ $(n=108,64 \%)$. There were 18 patients $(11 \%)$ who initially presented with thin $(\leq 1 \mathrm{~mm})$ melanomas. There were more ulcerated primary tumors than non-ulcerated primaries although there were a large number of patients who were missing data on ulcerationagain data on ulceration was not part of the AJCC staging system until 2002. The median number of examined nodes was 22 (range $=5-123$, mean=26.1). The median number of positive nodes was $2($ range $=1-55$, mean $=4.0)$. The largest group of patients $(n=76$, $45 \%$ ) were categorized as $\mathrm{N} 1$, while $39 \%$ and $15 \%$ were $\mathrm{N} 2$ and $\mathrm{N} 3$, respectively.

According to previous work, patients were assigned to groups based on LNR.

The majority of patients were in group $1(\mathrm{LNR} \leq 10 \%, \mathrm{n}=93,55 \%)$. There were 45 and 30 patients in groups $2(10-25 \%)$ and $3(>25 \%)$, respectively. Among patients with AJCC TNM N1 stage ( $\mathrm{n}=76$ ), the lowest lymph node ratio (group 1) had the most patients $(\mathrm{n}=37,49 \%)$. There were 29 patients (38\%) in group 2 and 10 patients in group 3 (Figure 1). For patients with N2 stage disease $(n=66)$, there were 38 patients in ratio 
group 1 (58\%), 15 in group 2 (23\%), and 13 patients (20\%) in the highest LNR group.

Finally, for patients with N3 regional disease $(n=26)$, there were 18 patients $(69 \%)$ with a ratio $\leq 10 \%$. (These 18 patients had a median of 54 lymph nodes removed, with a maximum of 123). In group 2, there was one patient, while in group 3, there were seven patients $(27 \%)$.

The median survival of the entire series was 34 months (95\% CI 29-44) with $36 \%$ (95\% CI 28\%-44\%) of patients surviving at least 5 years. Using univariate analysis, age, sex, year of surgery, tumor thickness, and ulceration were not significant predictors of survival (Table 2). One of the strongest predictors of overall survival (OS) was LNR $>25 \%(\mathrm{HR}=3.08, \mathrm{p}<0.01)$. A LNR between 10 and $25 \%$ was also an important predictor of survival (Table 2). Other factors that were significant on univariate analysis were type of treatment, number of positive nodes, and LN basin dissected (patients who had LND for cervical metastases had decreased survival compared to those with axillary metastases.) The total number of examined or excised lymph nodes was not an important factor for survival $(\mathrm{HR}=1.0$ per node, $\mathrm{p}=0.83)$. Finally, we saw a borderline significant difference in survival depending on whether the indication for lymph node dissection was performed for a positive sentinel node compared to those who underwent dissection for a clinically positive lymph node basin $(\mathrm{p}=0.08)$, as well as in primary sites with lower hazards in patients with either trunk or extremity versus head/neck $(p=0.10, p=0.09$ respectively.) 
Of the 3 different multivariate models concerning LNR and total number positive nodes variables (namely: both LNR and total number positive nodes in the model; LNR alone; total number positive nodes alone; these three models with the other variables as described in methods), the model with LNR alone had the smallest AIC (Akaike information criterion: $833.6,830.2$, and 836.2 , respectively). This multivariate analysis showed that the only factors that were predictive of overall survival were LNR and type of treatment. Those with a LNR $>25 \%$ had a 4 -fold higher risk of dying compared to those with a LNR $<10 \%(\mathrm{p}<0.01)$. Type of treatment is globally significant, but looking at the individual levels show that of the four types of treatment compared to none/unknown, only chemotherapy medications had an even marginally significant hazard ratio $(H . R .=1.90, p=0.08) . \mathrm{LN}$ basin was not a globally significant factor in the model $(p=0.20)$, however, those with more than one nodal basin involved had twice the risk of dying $(H . R .=2.12, p=0.05)$ compared to axillary. When included in the model with LNR, as an alternate analysis, the total number of positive nodes was not significant, $p=0.75$, although this is somewhat obscured by the fact that this value is used to compute (and may be collinear with) LNR. LNR was still significant, $\mathrm{p}<0.024$.)

In terms of median and 5-year survival (Figure 2, Table 3) of patients by LNR, we found a significant difference among the 3 LNR groups. Patients in group 1 had a median survival of 77 months $(95 \%$ CI $43, \infty)$ with estimated 5-year survival of $52 \%$ (95\% CI 40\%-63\%). Patients in groups 2 and 3 had 5-year survivals of $24 \%$ and $0 \%$, respectively. The separation of the 3 survival curves was persistent throughout the entire 
course of follow-up. We also looked at median and overall survival based on number of positive nodes (AJCC N stage N1, N2, and N3); these are presented in Figure 3. As you can see from this figure, there is a difference in survival with N1 patients surviving a median of 52 months compared to 26 months for those with N3 disease (Table 3). However, the survival curves of the N1 and N2 patients do not have a lot of separation from each other like we see with the plots for LNR. Using AIC, we were able to determine that the LNR model provided a better fit for overall survival than AJCC N Stage. 


\section{DISCUSSION}

1

Melanoma has been increasing in frequency for the past several years and has now become the seventh most common malignancy diagnosed in the United States. Patients with stage III melanoma cover a wide range of disease spectrums from those with microscopically positive lymph nodes found with sentinel node mapping and a nonulcerated primary (Stage IIIa) who have an estimated 5-year survival rate of $\sim 70 \%$ according to AJCC data ${ }^{2}$ down to patients with ulcerated primary tumors and clinically positive nodal basins with more than 3 nodes involved. These patients have estimated 5| year survivals down to $13 \% \frac{12}{2}$.

Deleted:

This vast heterogeneity in survival for stage III patients causes limitations in the applicability of the current staging system in terms of making treatment decisions and stratifying patients for adjuvant therapy and clinical trials. Currently the only treatment approved by the FDA in the US for the adjuvant treatment of patients with stage III melanoma is Interferon-alpha-2b. Multiple studies have shown this treatment to increase relapse free survival but there has been little impact on overall survival. Most of these studies were completed at a time when the majority of patients diagnosed with stage III disease had macroscopic or clinically positive disease. Clearly these patients are at higher risk and may benefit more from treatment. However, the patients diagnosed with microscopic disease on sentinel node biopsy or with minimal lymph node involvement probably represent a lower risk group. For example, in the recently completed Sunbelt Melanoma Trial ${ }^{13}$, patients with one positive lymph node by sentinel node biopsy were 
randomized to completion LND versus LND plus one year of standard adjuvant therapy with Interferon-a. This trial showed no difference in disease-free or overall survival ${ }^{12}$ for this group of patients that could be considered lower risk.

Therefore, it is imperative that we find more reliable prognostic factors which could be of greater clinical benefit in providing patients with the highest chance of cure. One prognostic factor which has become more prevalent in patients with lymph node positive cancer is that of the ratio of metastatic to examined nodes or LNR (also called Nratio). There are several examples in the literature which have demonstrated the importance of LNR in determining survival. In a gastric cancer study, Bando et al divided 650 patients into four groups based on LNR. These ratios were 0,0 to 10,10 to 25 , and more than 25 ; the 5-year survival rates were $86 \%, 68 \%, 35 \%$, and $16 \%$, respectively, and LNR was an independent prognostic factor ${ }^{8}$. Another recent study by Rossi et al, was the first to demonstrate the prognostic significance of LNR in patients with stage III melanoma ${ }^{5}$. In this study, patients were divided into 3 groups based on LNR of $\leq 10 \%, 10-\leq 25 \%$, and $>25 \%$. These authors also demonstrated that LNR was an independent prognostic factor for survival. They also found that TNM stage was the strongest independent predictor of survival ${ }^{5}$. The only primary tumor dependent factor which was significant on multivariate analysis was tumor thickness measured as a continuous variable.

In our study, we too found that LNR was an independent predictor of overall survival. In fact, we did not determine any variables related to the primary tumor to be 
predictive of overall survival. Our study was slightly different from that of Rossi et al in that we categorized tumor thickness into 4 groups as it is in the current AJCC classification. This is similar to findings of the group at John Wayne Cancer Institute who found that the most important factors for survival in patients with stage 3 melanoma are those found at the time of lymph node dissection—number of positive nodes and whether lymph nodes were clinically palpable ${ }^{4}$. Tumor thickness as a primary related factor was still significant but less so in their model. The majority of patients in our $\underline{\text { series were treated before sentinel lymph node biopsy became standard of care in }}$ melanoma treatment. Therefore, only $61(31 \%)$ of patients underwent completion lymph node dissection for a positive sentinel lymph node. We did find that the indication for lymph node dissection was of borderline significance on univariate analysis but lost this trend on multivariate analysis $(\mathrm{p}=0,32)$. We acknowledge that in the current era, the majority of regional lymph node dissections are performed for positive sentinel lymph nodes and that the current study may not be completely applicable. We are currently in the process of analyzing a more current and larger database to examine this phenomenon. The most comprehensive analysis to date on the impact of LNR in melanoma was published recently by the Melanoma Institute of Australia ${ }^{10}$.In this analysis, the authors used the previous cutoffs set out by Rossi et al $(<10 \%, 10-25 \%$, and $>25 \%)$ and confirmed the importance of LNR in a large melanoma population $(\mathrm{n}=1514)$ with $\underline{\text { standardized surgical quality. Furthermore, they showed that LNR also allowed for }}$ $\underline{\text { substaging of the AJCC N3 patients. There was no separation of patients in this analysis }}$ 
based on indication for regional LND even though their series spanned the era of

$\underline{\text { therapeutic versus completion dissection. }}$

In a recent analysis of the SEER database for patients undergoing therapeutic

LND for melanoma, Xing et al evaluated the importance of LNR by examining a LNR

threshold above which disease-specific survival significantly decreases. They determined these thresholds to by $7 \%, 13 \%$, and $18 \%$ for neck, axillary, and inguinal regions, respectively ${ }^{6}$. In their multivariate analysis, patients who had a LNR less than the threshold had a 50\% reduction in their hazard ratio for disease-specific death $(\mathrm{p}<0.001)$.

${ }^{6}$. One of the limitations of this study is that there is a relatively short median follow-up time of 3 years; additionally, the SEER database does not have data on adjuvant treatment.

Some people may also be troubled by the fact that a primary tumor characteristic such as ulceration was not a significant factor for survival. In the study of Balch et al of the AJCC database, these authors found 5 factors which were independent predictors of survival in 1200 patients with lymph node metastases. These included the number of metastatic nodes, the tumor burden (micro- vs. macroscopic), ulceration of the primary, age, and the site of the primary tumor ${ }^{2}$. However, since the majority of patients in this study underwent lymph node dissection for clinically positive lymph node basins at some point distant from their primary tumor resection, it would seem intuitive that survival should be influenced primarily by characteristics of the stage III disease, such as the number of positive nodes and lymph node ratio. As our group has pointed out 
previously ${ }_{\mathrm{w}}^{14}$, the Balch study is somewhat flawed by the fact that survival was measured from the date of diagnosis of the primary tumor instead of from the date of lymph node dissection. For this reason, characteristics of the primary, such as ulceration probably played a bigger role in their analysis. We also recognize that approximately one-third of patients did not have data regarding ulceration status in our series. Unfortunately, a large proportion of our patients are referred from outside dermatologists after undergoing primary removal and data regarding ulceration was not universally reported prior to 2002 when it was first made part of the AJCC staging system. It would be very difficult to go back and analyze all of these specimens for ulceration. We acknowledge that this is a $\underline{\text { limitation in our study. }}$

Finally, in looking at survival curves based on LNR group vs. N category, we see that there is a much better discrimination between patients using LNR than with $\mathrm{N}$ stage (Figures 2 and 3). As the LNR increased from $\leq 10 \%$ to over $25 \%$, the 5 -year survival correspondingly dropped off from $52 \%$ (95\% CI $40 \%-63 \%)$ to $24 \%$ (95\% CI $11 \%-40 \%$ ) to $0 \%$. However, 5-year survivals by $\mathrm{N}$ stage were similar for N1 and N2 disease (48\% (95\% CI 35\%-60\%) and 44\%, (95\% CI 36\%-60\%), respectively). Therefore, we feel that the ratio of positive to examined lymph nodes (LNR) should play a more prominent role in the staging system for melanoma and serve as important stratification for patients undergoing adjuvant therapy for stage III melanoma. We hope to further validate these findings in larger patient datasets in the future. 


\section{REFERENCES}

1. Geller AC, Miller DR, Annas GD et al. Melanoma incidence and mortality among US whites, 1969-1999. JAMA. 2002;288:1719-1720.

2. Balch CM, Soong SJ, Gershenwald JE et al. Prognostic factors analysis of 17,600 melanoma patients: validation of the American Joint Committee on Cancer melanoma staging system. J Clin Oncol. 2001;19:3622-3634.

3. Calabro A, Singletary SE, Balch CM. Patterns of relapse in 1001 consecutive patients with melanoma nodal metastases. Arch Surg. 1989;124:1051-1055.

4. Morton DL, Wanek L, Nizze JA et al. Improved long-term survival after lymphadenectomy of melanoma metastatic to regional nodes. Analysis of prognostic factors in 1134 patients from the John Wayne Cancer Clinic. Ann Surg. 1991;214:491-499.

5. Rossi CR, Mocellin S, Pasquali S et al. N-ratio: a novel independent prognostic factor for patients with stage-III cutaneous melanoma. Ann Surg Oncol. 2008;15:310-315.

6. Xing Y, Badgwell BD, Ross MI et al. Lymph node ratio predicts disease-specific survival in melanoma patients. Cancer. 2009;115:2505-2513. 
7. Inoue $\mathrm{K}$, Nakane $\mathrm{Y}$, Iiyama $\mathrm{H}$ et al. The superiority of ratio-based lymph node staging in gastric carcinoma. Ann Surg Oncol. 2002; 9:27-34.

8. Sierra A, Regueira FM, Hernandez-Lizoain JL et al. Role of the extended

lymphadenectomy in gastric cancer surgery: experience in a single institution.

Ann Surg Oncol. 2003; 10:219-26.

9. Bhatti I, Peacock O, Awan AK, et al. Lymph node ratio versus number of affected

Deleted: . 8. Stein HJ, Feith M, Siewert JR. Cancer of the

esophagogastric junction. Surg Oncol. 2000;9:35-41.II

lymph nodes as predictors of survival for resected pancreatic adenocarcinoma.

World J Surg. 2010; 34(4):768-75.

Deleted: St CB, Rago C, Velculescu V et al. Genes expressed in human tumor endothelium. Science. 2000;289:11971202.

10.Spillane AJ, Chenug BLH, Winstanley J et al. Lymph note ratio provides prognostic

information in addition to American Joint Committee on Cancer $\mathrm{N}$ stage in

patients with melanoma, even if quality surgery is standardized. Ann Surg. 2011;

253:109-15.

11. Balch CM, Buzaid AC, Soong SJ et al. Final version of the American Joint

Deleted: 10

Committee on Cancer staging system for cutaneous melanoma. J Clin Oncol.

2001;19:3635-3648.

12. Akaike H. A new look at the statistical model identification. IEEE Transactions on

Deleted: 11

Automatic Control 1974; 19 (6): 716-723 
13. McMasters KM, Ross MI, Reintgen DS et al. Final results of the Sunbelt

Melanoma Trial. J Clin.Oncol. 2008.

Ref Type: Abstract

14. Berd D, Mastrangelo MJ, Sato T. Calculation of survival of patients with stage III

Deleted: 13 melanoma. J Clin Oncol. 2005;23:9427. 


\section{FIGURE LEGENDS:}

Figure 1: Demonstrates the breakdown of patients in AJCC N1, N2, and N3 categories by the corresponding lymph node ratio.

Figure 2: Kaplan-Meier survival curve which demonstrates overall survival as determined by lymph node ratio $(<10 \%, 10-25 \%$, and $>25 \%)$ with a clear decrement in survival with increasing ratio. This relationship is highly statistically significant $(\mathrm{p}<0.0001)$.

Figure 3: Kaplan-Meier curve demonstrating the relationship between survival and AJCC $\mathrm{N}$ stage (N1, N2, and N3). Here you can see that there is a statistically significant relationship between increasing $\mathrm{N}$ stage and worsening survival. However, there is not as much separation of curves as demonstrated in Figure 2. 
Berger-21 